\title{
ESPACIO PÚBLICO EN CIUDADES PRIVADAS Centralidades emergentes y su impacto en las periferias metropolitanas de Latinoamérica
}

\author{
Public Space in private cities \\ Emerging centralities and their impact in metropolitan peripheries \\ of Latin America
}

\author{
Vidal-Koppmann, Sonia; \\ Consejo Nacional de Investigaciones Científicas y Técnicas (CONICET) / Universidad de Buenos Aires (UBA) \\ sonia.vidalk@gmail.com
}

\section{RESUMEN}

En el presente siglo, la dinámica socio-territorial de las regiones metropolitanas en América latina, muestra el impacto del urbanismo de mercado en el paisaje de las periferias urbanas. En este escenario, los límites entre los espacios públicos y privados se vuelven difusos, apareciendo nuevos formatos urbanos (barrios cerrados y ciudades privadas). Por ello, nos focalizaremos en el análisis de los espacios públicos dentro de ciudades privadas, específicamente en el diseño de sus áreas centrales. Aplicaremos el concepto de centralidades emergentes para diferenciarlas de las áreas centrales pre-existentes en las ciudades abiertas que configuran su entorno. Asimismo, consideraremos la dinámica de interacción entre dichos centros; y reflexionaremos sobre el desarrollo geográfico desigual y la fragmentación socio-territorial, que se va generando a medida que el urbanismo de mercado se vuelve hegemónico en la expansión de las periferias metropolitanas.

Palabras clave: regiones metropolitanas, urbanización privada, espacio público, centralidades emergentes. Bloque temático: espacio público y proyecto urbano en la metrópolis contemporánea

\section{ABSTRACT}

In this century, the socio-territorial dynamics of metropolitan regions in Latin America, shows the impact of market urbanism on the landscape of urban peripheries. In this scenario, the boundaries between public and private spaces become diffuse, with the appearance of new urban formats (closed neighborhoods and private cities). Therefore, we will focus on the analysis of public spaces within private cities, specifically in the design of their central areas. We will apply the concept of emerging centralities to differentiate them from the preexisting central areas in the open cities that make up their environment. We will consider the dynamics of interaction said centers; We will reflect on the unequal geographic development and the socio-territorial fragmentation that is generated as market urbanism becomes hegemonic in the expansion of the metropolitan peripheries.

Keywords: metropolitan regions, private urbanization, public space, emerging centralities.

Topic: public space and urban project in the contemporary metropolis 


\section{Introducción}

Las investigaciones acerca de las formas que adopta la expansión urbana a comienzos del siglo XXI ha dado lugar a numerosos trabajos. En particular, la dinámica socio-territorial de las regiones metropolitanas en América latina conlleva a observar el fenómeno del urbanismo de mercado como el "gran hacedor" de la morfología que adopta la urbanización, principalmente en las periferias. La localización de amplias fracciones de suelo rústico de muy baja densidad; la ampliación de infraestructuras para la movilidad y el transporte; la normativa laxa de ordenamiento territorial y la pujanza de los desarrolladores inmobiliarios, han favorecido la localización de proyectos de urbanizaciones privadas y de nodos de actividades terciarias con fuerte impronta territorial y ambiental.

Como objeto de análisis nos focalizaremos en el caso de la Región Metropolitana de Buenos Aires (RMBA), en donde más de 500 proyectos residenciales cerrados se van consolidando aceleradamente. Dentro de las distintas tipologías de diseño de estas urbanizaciones, sobresalen las ciudades privadas ${ }^{1}$ que ya suman doce proyectos con diferentes grados de consolidación. Las mismas se han emplazado en proximidad a los corredores viales de alta velocidad (cinco en total), en un radio de alrededor de $100 \mathrm{~km}$ del epicentro de la Ciudad de Buenos Aires.

Esta tipología de urbanización está contemplada en la normativa urbanística de la Provincia de Buenos Aires. Más precisamente en el Decreto-Ley 8912 promulgado en $1977^{2}$ que establecía las bases para el ordenamiento territorial a escala provincial. Bajo el subtítulo de "Urbanizaciones especiales" se sintetizan los componentes que debe contener el Plan Maestro para proyectar ciudades por parte de agentes privados.

Si bien muchas investigaciones dan cuenta de estas nuevas formas de urbanización (Prévot-Schapira, 2002; Janoschka, 2002; Borsdorf, 2003, entre otros); revisitar casos emblemáticos como Nordelta, Villa Nueva, Pilar del Este; etc.; a casi veinte años de su creación, permite observar las transformaciones operadas en la periferia metropolitana y los conflictos y tensiones emergentes entre "ciudades abiertas" y "ciudades cerradas". Como parte de este análisis, consideramos necesario estudiar la dinámica socio-espacial que se genera en los espacios públicos contenidos en ciudades privadas.

Es a partir de este punto y dando continuidad a los estudios sobre las transformaciones de las regiones metropolitanas, que orientamos los trabajos actuales hacia las áreas centrales, insertas en esta constelación de urbanizaciones privadas. Asimismo, consideramos que el estudio de estos casos plantea una confrontación entre dos tipos de urbanismo: público y privado. Y a su vez que estos dos tipos de intervención sobre los territorios periféricos profundizan el desarrollo geográfico desigual (Harvey, 2007; Smith, 2012) y la fragmentación socio-territorial (Santos, 2004).

Basándonos en la hipótesis de que estas áreas de centralidad no amplían el sistema de centros urbanos como núcleos complementarios, ni emulan a los espacios públicos de las ciudades abiertas; sostenemos que las mismas configuran un patrón de urbanismo fragmentado y dan lugar a la materialización de espacios con un desarrollo urbano desigual y selectivo para ciertos segmentos de la población metropolitana.

Por lo tanto, planteamos como objetivo central analizar y reflexionar acerca de los nuevos espacios públicos diseñados en las ciudades privadas, que han devenido en nuevas centralidades periféricas (centralidades emergentes), extendiendo los límites difusos de las regiones metropolitanas (Monclús, 1998).

La metodología implementada se sustenta en trabajos de investigación realizados previamente sobre las mutaciones socio-territoriales de grandes regiones urbanas de América latina (Vidal-Koppmann, 2015),

\footnotetext{
${ }^{1}$ Las ciudades privadas son conjuntos de barrios cerrados cuyo diseño obedece a un Master Plan que contempla el diseño de espacios públicos, equipamientos y servicios y vialidades. Ver Vidal-Koppmann, 2014.

2 Decreto-ley promulgado durante la dictadura (1976-1983) y aún vigente.
} 
tomando como recorte geográfico las centralidades metropolitanas en la región de Buenos Aires. A partir de dichos trabajos se profundizó el análisis, acerca de las características y el emplazamiento de las ciudades cerradas; teniendo en cuenta las estrategias de los emprendedores inmobiliarios para su reproducción (VidalKoppmann, 2014).

En segundo lugar, se trabajó sobre la temática de la accesibilidad y la movilidad metropolitana en relación con las nuevas centralidades de estos núcleos urbanos (Vidal-Koppmann, 2018a). Esta línea apuntó a describir dos circuitos yuxtapuestos en la periferia de la RMBA. Por un lado, el de las localidades suburbanas conectadas por los sistemas de transporte público y habitadas por sectores de ingresos medios y medio-bajos; y por otro, el de las urbanizaciones privadas de las élites, vinculadas con las centralidades emergentes mediante la red de autopistas y el uso intensivo del automóvil.

$\mathrm{Y}$, en tercer término, desarrollamos una línea de trabajo acerca de la interacción entre ciudades privadas y ciudades abiertas, entendiendo que existe una dialéctica espacial y una disputa por los territorios periféricos que amplía la brecha de la polarización urbana (Svampa, 2005) y que constituye un desafío para la planificación y el ordenamiento territorial.

\section{Expansión, dispersión urbana y movilidad en la Región Metropolitana de Buenos Aires}

La dinámica de expansión acelerada de las regiones metropolitanas en Latinoamérica, ha puesto en evidencia la transición hacia un nuevo paradigma, donde la fragmentación socio-territorial de las periferias (De Mattos, 2010) y el desarrollo geográfico desigual (Harvey, 2007) conforman un patrón común. Esta condición es inherente a todas ellas, a pesar de las singularidades socioespaciales de cada país.

La región metropolitana de Buenos Aires (RMBA), la segunda en cantidad de población de Sudamérica, participa de estas características, mostrando en las últimas tres décadas la dinámica pujante de una lógica capitalista de intervención en los territorios y las deficiencias de un marco de ordenamiento urbano-territorial, que propone alternativas de planificación y gestión, con posterioridad a las transformaciones.

Como consecuencia de estos fenómenos en paralelo, pero con diferentes velocidades, pueden visualizarse en ella, espacios altamente polarizados donde conviven cuñas de riqueza ${ }^{3}$ en vecindad con bolsones de pobreza (Vidal-Koppmann, 2012); y donde las localidades urbanas consolidadas a lo largo de décadas por un espontáneo proceso de suburbanización (Torres, 1993; Chiozza, 2000; Hardoy y Gutman, 2007), se confrontan con nuevas urbanizaciones de carácter privado producto de la intervención del mercado inmobiliario (Figura 1).

\footnotetext{
${ }^{3}$ A lo largo de los corredores viales metropolitanos.
} 


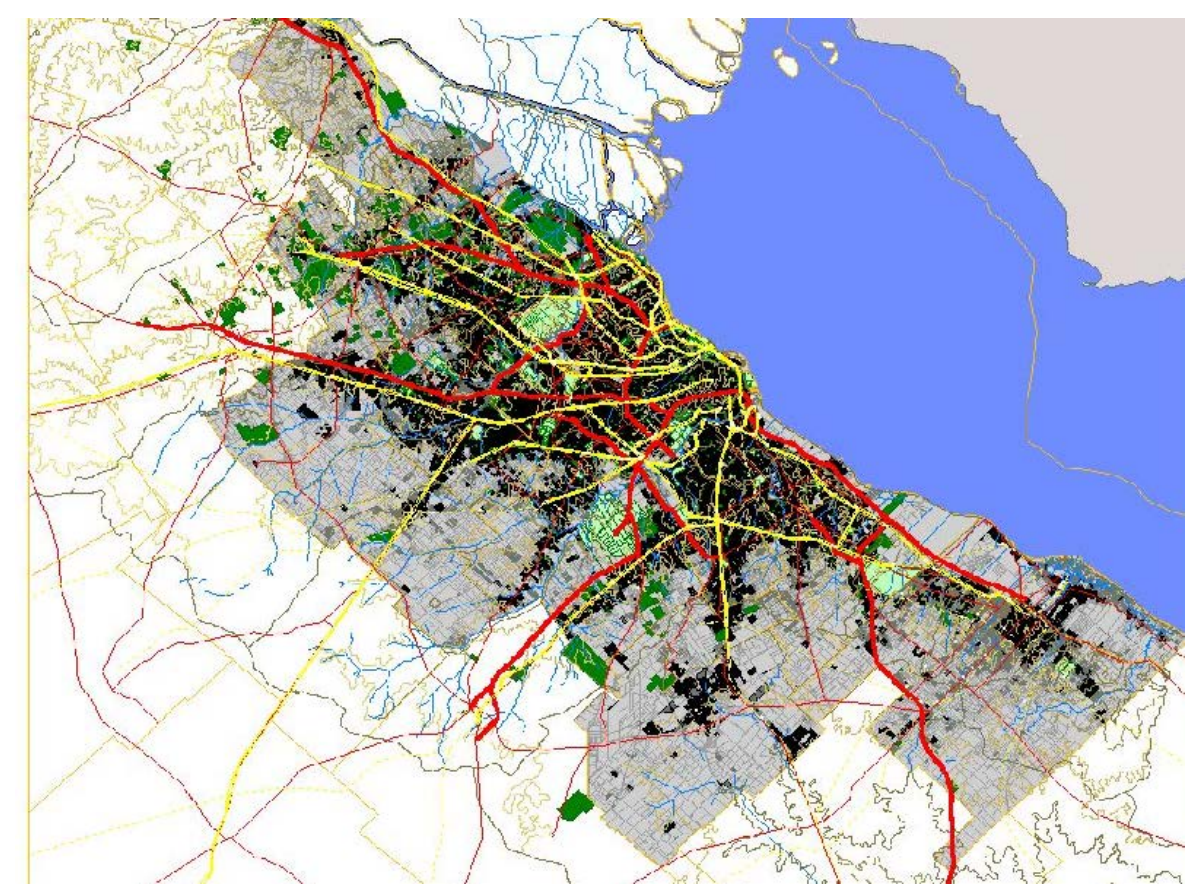

Figura 1. Fuente: Centro de Información Metropolitana, FADU-UBA, 2015

Obviamente ambos modelos de suburbanización coexisten en forma desintegrada (Vecslir y Ciccolella, 2012), tanto en la escala urbana como en la escala metropolitana, y remarcan la falta de un ordenamiento territorial (Mignaqui, 2012) que contemple de manera conjunta la planificación de usos del suelo con la planificación de infraestructuras -en particular, de movilidad y comunicaciones- y de equipamientos básicos.

El territorio "quebrado" (Muñoz, 2008) y estructurado en función de archipiélagos urbanos periféricos, presenta características de hibridez en una interfase urbano-rural poco definida (Dematteis, 1998) y menos aun incorporada en una planificación estratégica, que permita controlar y gestionar los procesos de expansión metropolitanos.

Estos escenarios representan nuevos desafíos para el ordenamiento territorial, tales como la consolidación de áreas de centralidad (básicamente concentradoras de usos comerciales, de servicios y de espacios de amenidades) por fuera de las localidades pre-existentes, y como respuesta a una nueva organización del uso del suelo residencial en aglomerados de barrios cerrados y ciudades privadas. Cabe destacar que estas nuevas centralidades no obedecen a un proyecto urbanístico de mejora y revitalización de zonas periféricas, tal como fuera el modelo de Barcelona (Capel, 2011). En el caso de Buenos Aires no existe la voluntad política de recualificar y/o potenciar las áreas de centralidad en las localidades de la periferia; al contrario, se deja en manos del urbanismo privado el diseño de centralidades, sin una lógica de ordenamiento territorial.

Así se diseñan áreas centrales en las ciudades privadas para uso casi exclusivo de sus habitantes. Si bien en las descripciones de los proyectos urbanísticos, se menciona que estos centros ampliarán la oferta de equipamientos terciarios de su entorno, el emplazamiento de los mismos y la falta de medios públicos de transporte conforman una barrera para su uso, reforzando la fragmentación socio-territorial.

Por otra parte, puede afirmarse que la profundización de dicha fragmentación está en relación directa con la expansión acelerada y sin límites y con las políticas públicas de ordenamiento que mantienen la centralidad de Buenos Aires y acrecientan su área de influencia, mediadas por las tecnologías de comunicación.

En efecto, la evolución del crecimiento de la RMBA, se generó a partir de extensos suburbios (loteos populares y auto-construcción) a lo largo de la traza ferroviaria en la primera mitad del siglo XX (Scobbie, 1977); y sigue 
creciendo territorialmente en la actualidad, apoyándose en sistemas de autopistas de alta velocidad y transporte automotor público y privado. De tal forma que la expansión presenta como características:

a) La incorporación de fragmentos urbanos discontinuos.

b) La promoción de ciudades privadas que ostentan el estatus de localidades.

c) La generación de una estructura reticular que articula centros urbanos independientemente de sus jerarquías.

d) El desdibujamiento de los límites inter-jurisdiccionales.

e) La incorporación del mercado como agente de planificación.

Desde los inicios de la década de los años 90, dicha expansión metropolitana incentivada por la extensión de las infraestructuras viales y los sistemas de comunicación y transporte, se ha desarrollado en paralelo con procesos de crecimiento urbano liderados por el mercado inmobiliario. En esta instancia, los protagonistas son los agentes privados, quienes van extendiendo los bordes del periurbano, con criterios poco compatibles con una planificación racional.

Dentro de este contexto, se observa el surgimiento de localidades privadas; la aproximación y fusión entre emprendimientos urbanos de grandes extensiones; los crecimientos lineales por sumatoria de urbanizaciones cerradas a lo largo de autopistas, y la concentración de equipamientos de envergadura (comercio y servicios) configurando nuevas áreas de centralidad.

Mientras tanto, la planificación estatal a diferentes escalas, no se adelanta a la problemática socio-territorial. Se buscan soluciones a la expansión metropolitana, cuando ésta ya se ha traducido en conflictos territoriales y ambientales difíciles de controlar. A la condición multi-escalar, multi-actoral y multi-sectorial de la RMBA, hay que adicionarle la falta de una autoridad metropolitana y de un plan estratégico integral. Si se tiene en cuenta que esta región de $13.934 \mathrm{~km}^{2}$ y más de 15 millones de habitantes (14.819.137 en el último censo nacional, INDEC 2010) está conformada por 41 municipios autárquicos y la Ciudad Autónoma de Buenos Aires, se puede comprender la complejidad de la situación.

\section{2. Áreas de centralidad en entornos privados ¿un nuevo concepto de espacio público?}

La cuestión de las centralidades emergentes en la región analizada nos lleva a destacar la presencia de dos patrones urbanísticos, que se replican en los municipios bonaerenses y que se encuentran directamente vinculados con el crecimiento de las formas de urbanización privada. Ambos patrones contrastan con las áreas centrales de las ciudades abiertas que se localizan en las cuatro coronas de municipios del área de influencia de la Ciudad de Buenos Aires.

En este sentido, los centros tradicionales de las ciudades cabecera y los subcentros de las localidades menores presentan una estructura de usos y ocupación del suelo muy semejante, siempre alrededor de una plaza fundacional y según un trazado en damero. Esta morfología urbana difiere sustancialmente de los nuevos modelos de centralidad que han aparecido en las regiones metropolitanas (Abba, 2010). Por este motivo, cuando nos referimos a las centralidades emergentes, hacemos alusión a dos tipos de agrupamientos de comercio y servicios: los polígonos de actividades terciarias localizados en entornos semi-rurales y próximos a vialidades de alta velocidad; y las áreas centrales contenidas en las ciudades privadas.

El primero de los patrones mencionado y observado en la RMBA, posee una estrecha relación con el fenómeno de crecimiento y concentración de urbanizaciones cerradas en zonas semi-rurales. Puede afirmarse que este fenómeno ha sido uno de los factores de mayor peso para el surgimiento de centros comerciales, malls, hipermercados, centros de amenidades, etc. y vastas superficies de estacionamiento de vehículos particulares. 
La posibilidad de contar con amplias fracciones de tierra en las zonas más alejadas de la periferia, a muy bajo costo, fue una oportunidad inmejorable para los negocios inmobiliarios liderados por grandes empresas desarrolladoras. Estas favorables condiciones financieras unidas a la carencia de una planificación metropolitana, facilitaron que los proyectos de estos polígonos de actividades terciarias fueran consolidándose a través del tiempo ${ }^{4}$. El nuevo modelo de ordenamiento urbano-territorial pone en evidencia la concentración de inversiones privadas especulativas (De Mattos, 2015).

Es preciso señalar que, en la mayoría de los casos, la localización de actividades urbanas en áreas semirurales ha requerido de ordenanzas de excepción a las zonificaciones vigentes, alterando el ordenamiento territorial. En los corredores Norte (Acceso Norte - AU Panamericana - ramales Tigre, Pilar y Escobar); Oeste (Acceso Oeste-Ruta Nacional no 7) ; Sudoeste (AU Ricchieri - AU Ezeiza-Cañuelas) y Sur (AU Buenos AiresLa Plata) se puede observar el proceso de suburbanización insular relacionado con el uso intensivo del automóvil (Blanco y San Cristóbal, 2012) y con la aparición de estas nuevas centralidades (Figura 2).

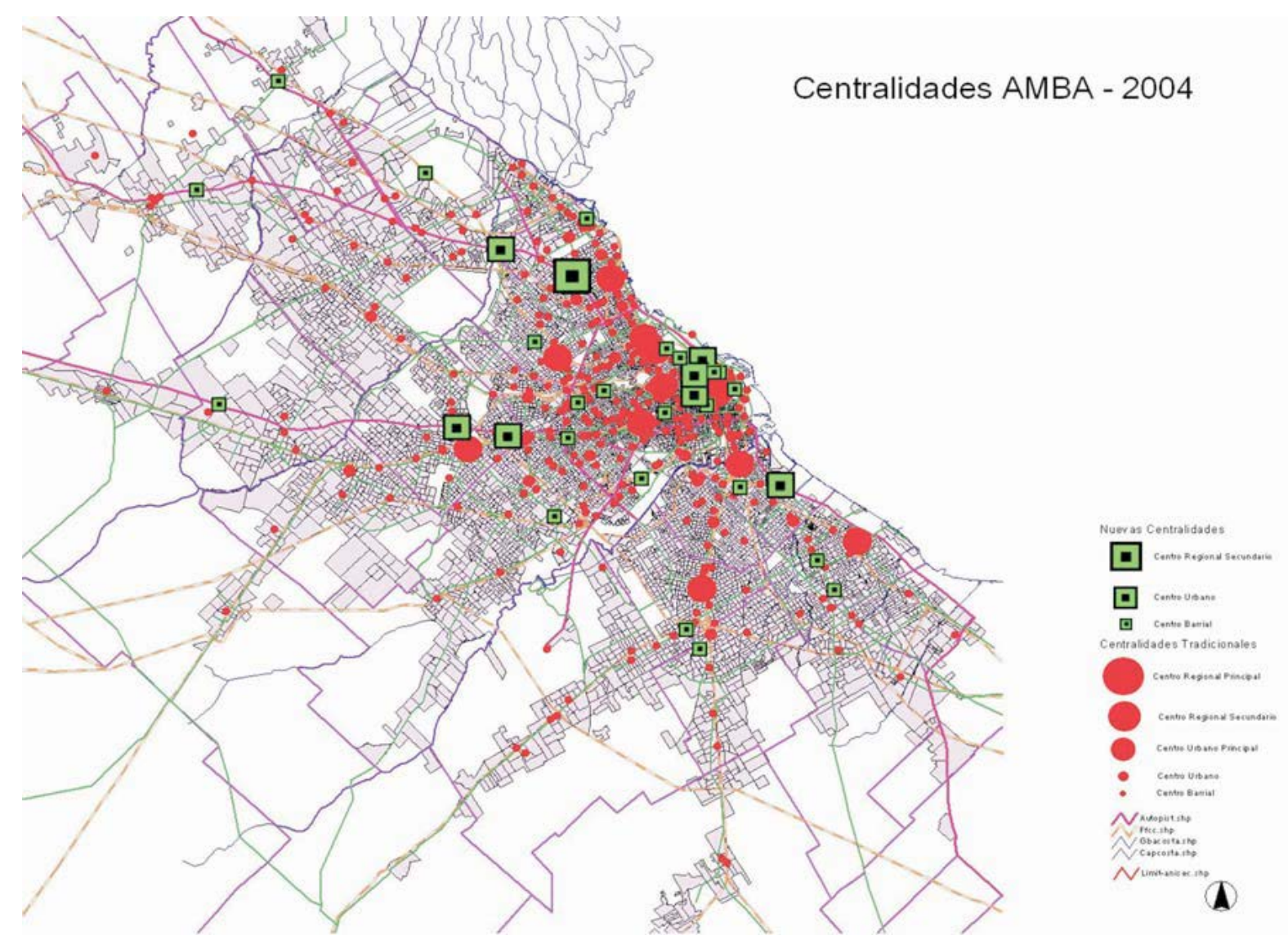

Figura 2. Centralidades tradicionales y emergentes. Fuente: Lineamientos estratégicos para la región Metropolitana de Buenos Aires, 2007.

Cabe agregar que estos emprendimientos no aumentan las superficies destinadas a espacios públicos, porque si bien han sido destinados para el uso de los ciudadanos son espacios gestionados y explotados por actores privados; y por ende, se puede ejercer una admisión selectiva de usuarios. En estos espacios es difícil concebir alguna expresión masiva espontánea, ni otro tipo de actividades que no sean las vinculadas al consumo y las amenidades (Duhau y Giglia, 2008).

${ }^{4}$ El emplazamiento de malls, home-centers, centros de amenidades, etc., en la periferia de la RMBA comienza a observarse a partir de los años '90. 
Por otra parte, hay que remarcar que de por sí solo un hipermercado o un centro comercial en la periferia metropolitana, no posee suficiente entidad como para devenir en un área de nueva centralidad. Aunque coincidimos con Vecslir (2011), al afirmar que cuando en un mismo territorio van emergiendo otras actividades asociadas (hoteles de alta gama, edificios corporativos, bancos, establecimientos educativos privados, etc.), el conjunto debería ser considerado como una subcentralidad que presenta una tipología urbanística diferente a la tradicional.

El segundo patrón de nueva centralidad señalado precedentemente, se encuentra asociado a las ciudades privadas, que también se están consolidando dentro de la región. Algunas de ellas ya cuentan con una población permanente considerable ${ }^{5}$. Bajo este formato, los usos de suelo comerciales y de servicios se concentran en una incipiente área central, destinada a satisfacer la demanda de los barrios cerrados que integran las mismas. Sobre este segundo modelo de centralidad, nos interesa enfocar nuestro análisis ya que como lo señalamos precedentemente, en la RMBA se han erigido una docena de ciudades privadas (VidalKoppmann, 2018b), que en la actualidad presentan diferentes etapas de ejecución (Cuadro 1).

\begin{tabular}{|c|c|c|c|c|}
\hline PROYECTO & MUNICIPIO & BARRIOS & POBLACIÓN PROY. & OBSERVACIONES \\
\hline Nordelta & Tigre / norte & $\begin{array}{l}23 \text { + área central + } \\
\text { colegios + c. salud } \\
\text { +universidad }\end{array}$ & 130.000 hab. & $\begin{array}{l}90 \% \text { del predio son } \\
\text { humedales. Salida al } \\
\text { río Luján }\end{array}$ \\
\hline Villanueva & Tigre / norte & $\begin{array}{l}11+\text { centro comercial } \\
\text { y de servicios }\end{array}$ & 45.000 hab. & $\begin{array}{l}\text { Laguna + salida al río } \\
\text { Luján }\end{array}$ \\
\hline Estancias del Pilar & Pilar / noroeste & $\begin{array}{l}5+3 \text { clubes } \\
\text { temáticos }+ \text { área } \\
\text { central (El Pueblo) }\end{array}$ & $\begin{array}{l}8.000 \text { hab. (Se redujo } \\
\text { la cifra inicial) }\end{array}$ & $\begin{array}{l}\text { Laguna artificial } \\
\text { aprovechando una } \\
\text { cava }\end{array}$ \\
\hline Pilar del Este & Pilar / noroeste & $\begin{array}{l}\text { + } 1 \text { en etapa de } \\
\text { construcción (13 } \\
\text { proyectados) }\end{array}$ & $\begin{array}{l}45.000 \text { en el proyecto } \\
\text { fundacional }\end{array}$ & $\begin{array}{l}\text { Cambio de empresa } \\
\text { desarrolladora }\end{array}$ \\
\hline Puertos del Lago & Escobar / noroeste & 20 + área central & 65.000 hab. & $\begin{array}{l}6 \mathrm{~km} \text { de costa sobre } \\
\text { el río Luján. Engloba } \\
\text { área de reserva } \\
\text { ecológica. }\end{array}$ \\
\hline San Sebastián & Pilar / noroeste & $\begin{array}{l}13+\text { espacios } \\
\text { deportivos, } \\
\text { comerciales, } \\
\text { educativos }\end{array}$ & $\begin{array}{l}25.000 \text { habitantes } \\
\text { (estimada) }\end{array}$ & $\begin{array}{l}\text { Barrios con } \\
\text { proximidad al río } \\
\text { Luján }\end{array}$ \\
\hline Puerto Palmas & Campana / norte & $\begin{array}{l}3+\text { c. comercial + } \\
\text { villa náutica + bahía }\end{array}$ & Sin datos & $\begin{array}{l}\text { Frente sobre Paraná } \\
\text { de las Palmas - C. } \\
\text { ecológica }\end{array}$ \\
\hline Puerto Trinidad & $\begin{array}{l}\text { Hudson (Berazategui) } \\
\text { / sur }\end{array}$ & $1+$ muelle y servicios & 80 familias & $\begin{array}{l}\text { Posee } 1 \mathrm{Km} \text { de costa } \\
\text { s/ Río de la Plata }\end{array}$ \\
\hline Colony Park & Tigre (Delta) / norte & $\begin{array}{l}\text { Isla privada con } 5 \\
\text { barrios }\end{array}$ & Sin datos & $\begin{array}{l}\text { Suspendido por } \\
\text { intervención judicial }\end{array}$ \\
\hline $\begin{array}{l}\text { Reserva Los } \\
\text { Cardales }\end{array}$ & Campana / norte & $\begin{array}{l}\text { Residencias + deptos. } \\
+ \text { hotel de lujo } \\
\text { (Sofitel) }\end{array}$ & Sin datos & $\begin{array}{l}\text { Reserva natural con } \\
\text { laguna de } 20 \text { has, }\end{array}$ \\
\hline Área 60 & Brandsen / sur & $\begin{array}{l}15+\text { c. comercial + } \\
\text { hotel internacional }\end{array}$ & 15.000 habitantes & $\begin{array}{l}\text { Sólo hay dos barrios } \\
\text { en construcción }\end{array}$ \\
\hline
\end{tabular}

${ }^{5}$ En Nordelta en el Municipio de Tigre se estima que habitan aproximadamente 35.000 personas. 


\begin{tabular}{|l|l|l|l|l|}
\hline El Principado & $\begin{array}{l}\text { San Vicente } / \\
\text { sudoeste }\end{array}$ & $\begin{array}{l}\text { Residencias uni y } \\
\text { multi-familiares + área } \\
\text { central y sectores } \\
\text { deportivos }\end{array}$ & $\begin{array}{l}\text { Sin datos } \\
\text { sectores } \\
\text { comunicados por } \\
\text { canales }\end{array}$ & $\begin{array}{l}\text { Diseño semi-abierto. } \\
\text { Parque industrial (60 } \\
\text { Has) }\end{array}$ \\
\hline Mirasoles & $\begin{array}{l}\text { E. Echeverría / } \\
\text { sudoeste }\end{array}$ & $\begin{array}{l}4+\text { área comercial + } \\
\text { parque industrial }\end{array}$ & Sin datos & $\begin{array}{l}\text { Apertura al Río de la } \\
\text { Plata (muelle) }\end{array}$ \\
\hline $\begin{array}{l}\text { Pampas Pueblo de } \\
\text { Hudson }\end{array}$ & Berazategui /sur & $\begin{array}{l}4+\text { Centro cívico } \\
\text { comercial }\end{array}$ & Sin datos, mix de \\
\hline
\end{tabular}

Cuadro 1. Ciudades privadas. Fuente: Elaboracón propia sobre la base de suplementos especializados, 2017.

En principio, debemos destacar que la localización de ciudades privadas en la periferia metropolitana no forma parte de una estrategia de ordenamiento territorial para descomprimir demográficamente la región; sino a la dinámica del mercado inmobiliario donde "hacer ciudad" equivale a un rentable negocio para multiplicar capitales financieros. El Plan Estratégico Territorial (Ministerio Federal de Planificación, 2011), en su volumen dedicado a la problemática de la urbanización (diagnóstico y proyecciones) destaca la necesidad de cualificar, potencializar y desarrollar los núcleos urbanos existentes, sin mencionar en absoluto la creación de nuevas urbanizaciones.

Y en segundo lugar, que si bien la RMBA cuenta en la actualidad con más de 500 urbanizaciones cerradas, solamente las que se han señalado en el cuadro precedente pueden encuadrarse en la categoría de ciudades privadas. Ello es debido, tanto a la propuesta del proyecto, como a la escala y a la dimensión demográfica de las mismas. La extensión territorial de estos emprendimientos va desde las 450 (Estancias del Pilar) a las 1400 hectáreas (Puertos del Lago). Por su capacidad locativa y por la complejidad de los equipamientos de abastecimiento, salud y educación, pueden asimilarse a núcleos urbanos pequeños (Área 60, San Sebastián) e intermedios (Nordelta, Villanueva, Puertos del Lago). Considerando estas variables básicas es posible caracterizar a estos megapro yectos como nuevas ciudades, estatus que desde el punto de vista políticoadministrativo ya ha alcanzado uno de los núcleos más antiguos. La ciudad de Nordelta, en el municipio de Tigre. es la primera localidad privada del país, según la Ordenanza Municipal 2524 y el Decreto 926, ambos promulgados en 2003.

Según lo señalado en la Introducción de este artículo, el Decreto-Ley 8912 de la Provincia de Buenos Aires menciona por primera vez el formato de "ciudad privada", englobándolo dentro del apartado correspondiente a Urbanizaciones especiales. Se destaca en el mismo que para su aprobación, estos mega-emprendimientos deberán contar con un Plan Maestro que contemple áreas residenciales, espacios públicos, infraestructura vial, servicios básicos y equipamientos comunitarios. En el plan mencionado también deberán ser consideradas las normas sobre uso, ocupación y subdivisión del suelo, destacando que la zonificación tendrá áreas destinadas a espacios verdes y públicos.

Del análisis de los esquemas de ordenamiento y planes directores de las ciudades privadas bonaerenses surge que, si bien existen zonas de uso público con concentración de equipamientos de comercio y servicio, la mayor dificultad consiste en acceder a los mismos desde las localidades vecinas. En este sentido, hay que destacar en primer lugar, que en estas ciudades hay accesos controlados por empresas de seguridad privada, que monitorean el ingreso de vehículos y peatones; y en segundo lugar, que para salvaguardar la tranquilidad de estos entornos privados, no se permite el acceso de medios de transporte público. Consecuentemente, se puede inferir que estos espacios destinados al uso público no son totalmente abiertos a toda la comunidad ya que de manera directa su acceso está mediado por sistemas de control; e indirecta, por la dificultad de llegar a ellos si no se es propietario de un vehículo. Solo en uno de estos conjuntos residenciales existe un empresa privada de transporte que circula por la avenida troncal del mismo. 


\section{Reflexiones finales}

En los apartados precedentes, se ha remarcado que la morfología de las nuevas áreas de centralidad, tanto las que obedecen a las demandas de los aglomerados de barrios cerrados de su entorno inmediato, como las diseñadas dentro de los planes maestros de las ciudades privadas, no responden a una necesidad de ampliación o de complementariedad con los centros de las localidades vecinas. Por el contrario, estos emprendimientos promueven circuitos funcionales selectivos, aumentando las condiciones de fragmentación territorial y segregación social. Sobre lo expuesto surgen nuevas preguntas y algunas reflexiones:

1. ¿Las nuevas centralidades permiten el libre acceso de todos los ciudadanos? La conectividad entre centros comerciales, multicines, hipermercados, polos gastronómicos, etc., está supeditada a los medios de transporte privado. Como bien lo señalan las investigaciones acerca de la movilidad urbana realizadas por Blanco (2005) y Blanco y San Cristóbal (2011) y las observaciones directas que realizamos sobre los corredores Norte y Sudoeste de la RMBA, la falta de medios de transporte público restringe el acceso. Asimismo, su localización en relación con bajadas de autopistas y cruces de arterias de alta velocidad, impiden al peatón llegar por sus propios medios. En el caso de las zonas comerciales de las ciudades privadas, la ausencia de transporte público que comunique el interior con el entorno, implica que solo los visitantes que posean un medio de transporte privado pueden acceder a ellas. Incluso esta situación motiva a que los empleados de los centros comerciales y/o locales de servicios deban utilizar empresas de mini-buses privados o transporte propio. Estas nuevas áreas de centralidad han sido diseñadas casi exclusivamente en función del uso intensivo del automóvil.

2. ¿Los servicios de seguridad privada condicionan o limitan las prácticas urbanas de los posibles usuarios? Tanto las áreas de centralidad externas a las zonas urbanizadas como los centros de las ciudades privadas se encuentran vigilados por cámaras, domos, personal privado y toda clase de dispositivos que intentan brindar a los usuarios la tranquilidad de no ser importunados con hechos delictivos fortuitos. Asimismo, como todos estos espacios son de uso público, pero de dominio privado, la total libertad de acceder a ellos está regida por un tácito derecho de admisión y de control social.

3. ¿Las centralidades emergentes aumentan la oferta y mejoran la calidad de los espacios públicos? En párrafos anteriores se expuso que la oferta de usos del suelo en estas nuevas áreas se encontraba limitada a rubros tales como moda, entretenimientos, gastronomía y servicios financieros o bancarios. Eventualmente en las ciudades privadas más consolidadas la oferta se extiende a centros de salud y de educación también de gestión privada; por lo tanto, la diversificación de equipamientos comunitarios y de servicios comparados con los que se encuentran localizados en las ciudades intermedias aledañas es bastante limitada. Las marcas globalizadas son las que ocupan la mayoría de estos espacios y están destinadas a determinados segmentos de población. En algunas ciudades privadas se han proyectado espacios para estudios y oficinas particulares, pero siempre en pequeña escala. En consecuencia, no es posible afirmar que haya una mejora relevante en cuanto a cantidad y calidad de servicios, que pueda trasladarse a una recualificación de la periferia metropolitana.

Para concluir deseamos volver sobre la principal pieza legal que ordena la creación de estas áreas, el Decretoley 8912/77 que reglamenta los usos del suelo y el ordenamiento territorial para la provincia analizada. Desde sus primeros párrafos se desprende que este tipo de urbanización privada debe estar debidamente justificada y que su localización en el territorio, tiene que estar en concordancia con los planes tanto de los municipios como de la región; sin embargo, en gran parte de los casos analizados parecería que estas dos premisas fundamentales no se han tenido en cuenta, dando lugar a la conveniencia e intereses de ciertos actores privados. Reiteramos, entonces, el desafío que tiene por delante el ordenamiento urbano-territorial y la necesidad de actualizar sus dispositivos y estrategias en función de ello. 


\section{BIBLIOGRAFÍA}

ABBA, A. (2010). Metrópolis Argentinas. Agenda política, institucionalidad y gestión de las aglomeraciones urbanas interjurisdiccionales. Buenos Aires: Ed. Café de las Ciudades.

BLANCO, J. (2005) Urbanización y movilidad: contradicciones bajo el modelo automóvil-intensivo. X Encontro de Geografos de América Latina, Sao Paulo, 4-10 marzo, (CD-Rom).

BLANCO, J. y SAN CRISTÓBAL, D. (2011). Restructuración de la red de autopistas y metropolización de Buenos Aires, Revista Iberoamericana de Urbanismo, (5), 73-88.

BORSDORF, A. (2003). ¿Cómo modelar el desarrollo y la dinámica de las ciudades latinoamericanas?. Revista EURE, (Santiago), 86, 37-49.

CAPEL, H. (2011). El modelo Barcelona: un examen crítico. Barcelona: Ediciones del Serbal.

CICCOLELLA, P. (2011). Metrópolis latinoamericanas: más allá de la globalización (1ª Edición). Quito: OLACCHI - Municipio Metropolitano de Quito.

CHIOZZA, E. (2000). La integración del Gran Buenos Aires. En ROMERO, J.L. y ROMERO, L. A. (Comp.) Buenos Aires. Historia de cuatro siglos. (411-434) Buenos Aires: Ed. Altamira.

DEMATTEIS, G. (1998). Suburbanización y periurbanización. Ciudades anglosajonas y ciudades latinas. En MONCLÚS, F. (Comp.) La ciudad dispersa. (17-36) Barcelona: CCCB.

DE MATTOS, C. (2004). Santiago de Chile de cara a la globalización ¿otra ciudad? En AGUILAR, A. (Coord.), Procesos metropolitanos y grandes ciudades. Dinámicas recientes en México y otros países, (19-52). México: H. Cámara de Diputados de la LIX Legislatura, Universidad Nacional Autónoma de México, Instituto de Geografía, Centro Regional de Investigaciones Multidisciplinarias, Consejo Nacional de Ciencia y Tecnología.

DE MATTOS, C. (2010). Globalización y metamorfosis urbana en América latina. Quito: OLACCHI.

DE MATTOS, C. (2015). Revolución urbana. Estado, mercado y capital en América Latina. Santiago de Chile: RiL Editores.

DUHAU, E. y GIGLIA, A. (2008). Las reglas del desorden: habitar la metrópolis. Ciudad de México: Siglo XXI Editores.

HARDOY, J. y GUTMAN, M. (2007). Buenos Aires 1536-2006. Buenos Aires: Ediciones Infinito.

HARVEY, D. (2007). Urbanismo y desigualdad social. 7ª. Ed. En español, Madrid: Siglo XXI.

JANOSCHKA, M. (2002). El nuevo modelo de ciudad latinoamericana. Revista EURE, (Santiago),85, 11-20.

MIGNAQUI, I. (2012). Planes y proyectos territoriales. Escenarios de la metrópolis planificada. Revista Iberoamericana de Urbanismo, (Madrid), 8, 121-137.

MUÑOZ, F. (2008). Urbanalización. Paisajes comunes, lugares globales. Barcelona: Editorial Gustavo Gili S.L. PRÉVOT-SCHAPIRA, M. (2002). Buenos Aires en los años 90: metropolización y desigualdad. Revista EURE, (Santiago),85, 31-50.

PROVINCIA DE BUENOS AIRES, SECRETARIA DE PLANEAMIENTO (1977). Decreto-ley 8912. Usos del Suelo y Ordenamiento Territorial. Buenos Aires: Autor.

REPÚBLICA ARGENTINA, MINISTERIO DE ECONOMÍA, INDEC (2010). Censo Nacional de Población, Hogares y Vivienda. Buenos Aires: Autor.

REPÚBLICA ARGENTINA, MINISTERIO FEDERAL DE PLANIFICACIÓN (2011). Plan Estratégico Territorial. Argentina Urbana. Buenos Aires: Autor.

SANTOS, M. (2004). A Natureça do Espaço. San Pablo: EDUSP.

SCOBIE, J. (1977). Buenos Aires del centro a los barrios. Buenos Aires: Ediciones del Solar - Hachette.

SMITH, N. (2012). La nueva frontera urbana. Madrid: Traficantes de sueños. 
SVAMPA, M. (2005). La brecha urbana. Buenos Aires: Ed. Capital Intelectual.

TORRES, H. (1993). El mapa social de Buenos Aires (1940-1990). Buenos Aires: Secretaría de Investigación y Posgrado, FADU-UBA.

VECSLIR, L. (2011). Nuevas centralidades del ocio y del consumo: ámbitos, modalidades e instrumentos de regulación de las grandes superficies comerciales en la Región Metropolitana de Buenos Aires. Revista Iberoamericana de Urbanismo, (Madrid), 5, 31-44.

VECSLIR, L. y CICCOLELLA, P. (2012). Transformaciones territoriales recientes y reestructuración metropolitana. Revista Iberoamericana de Urbanismo, (Madrid), 8, 1-7.

VIDAL-KOPPMANN, S. (2012). Movilidad y urbanizaciones privadas. En LIBERALI, A., VIDAL, S. y ORDUNA, M. (Directores) Movilidad y pobreza. Una prioridad en la agenda metropolitana (11-32) Buenos Aires: Ediciones CETAM/FADU,

VIDAL-KOPPMANN, S. (2014). Countries y barrios cerrados. Buenos Aires: Ed. Dunken.

VIDAL-KOPPMANN, S. (2015). Desarrollo desigual y combinado en la periferia metropolitana de Buenos Aires. Grandes emprendimientos privados y generación de cuñas de riqueza. En VIDAL-KOPPMANN, S. (Comp.) Metrópolis en mutación (83-101). Buenos Aires: Ed. Café de las Ciudades.

VIDAL-KOPPMANN, S. (2018 a). Ciudades privadas: a la búsqueda del paraíso ecológico. Estudio de casos en la Región Metropolitana de Buenos Aires. Teoría Socio Urbana, (Buenos Aires - Ciudad de México), 1, 91102.

VIDAL-KOPPMANN, S. (2018 b). Los conglomerados de urbanizaciones privadas: una nueva frontera para el ordenamiento urbano-territorial de la Región Metropolitana de Buenos Aires. En VIDAL-KOPPMANN, S. (Comp.) Dinámica socio-espacial de regiones metropolitanas. Claves para el análisis de un fenómeno complejo (119-134). Buenos Aires: Ed. IMHICIHU-CONICET. 